\title{
Investigation of histopathological effects after intra-arterial sugammadex injection in an experimental animal model
}

\author{
Kiraz $\mathrm{HA}^{1}$, Toman $\mathrm{H}^{1}$, Erbas $\mathrm{M}^{1}$, Yuyucu Karabulut $\mathrm{Y}^{2}$, Simsek $\mathrm{T}^{1}$, Hanci $\mathrm{V}^{3}$, Uzun $\mathrm{M}^{4}$ \\ Department of Anesthesiology and Reanimation, Faculty of Medicine, Canakkale Onsekiz Mart University, \\ Canakkale, Turkey dralikiraz@gmail.com
}

\begin{abstract}
PURPOSE: Intra-arterial injection of medications are related to mortality and morbidity. It was aimed to investigate the histopathological effects caused by two different intra-arterial doses of sugammadex, a new selective relaxant binding agent used to reverse the effects of steroidal systemic muscle relaxants used in surgeries carried out under general anesthesia.

METHODS: A total of 15 rabbits were randomly divided into 3 groups. The control group was given $1 \mathrm{~mL}$ saline bolus into the central artery of the ear. The other two groups (Group Sgdx2 and group Sgdx16) were given 2 and $16 \mathrm{mg} / \mathrm{kg}$ sugammadex, respectively, intra-arterially.

RESULTS: In control group, histopathological evaluation was in normal limits. The cross-sections belonging to Group Sgdx2 did not have any significant pathological change compared with control group $(p>0.05)$. Edema and single-cell necrosis were significantly higher in Group Sgdx16 when compared to control group $(p<0.05)$. In comparison to Group Sgdx2, single-cell necrosis was significantly higher in Group Sgdx16 ( $<<0.05)$.

CONCLUSION: Being broadly used in anesthesia, sugammadex should be administered with care because when given by intra-arterial route it may cause tissue damage and tissue loss and the patients' arteries should be carefully checked (Tab. 2, Fig. 3, Ref. 21). Text in PDF www.elis.sk.

KEY WORDS: dose, experimental, intra-arterial, rabbit, sugammadex.
\end{abstract}

\section{Introduction}

Accidental intra-arterial injections have very destructive effects on patients' lives $(1,2)$. Case reports about iatrogenic intraarterial injection of medications are available since the 1940s. The medications reported in this way and associated with mortality and morbidity are mainly sedation or medications used for general anesthesia. The incidence of intra-arterial injection and cannulation is known to be between $1 / 3440$ and 1/56000 (3). The intra-arterial injection of medications can cause acute and severe ischemia. Such situations are rarely observed and thus it is difficult to fully determine the correct incidence (4). After giving intra-venous forms of medications through the intra-arterial route local ischemia may develop in the artery which may possibly result in tissue necrosis. Mechanisms of intra-arterial medication injection and development of ischemia frequently include formation of medication crystals in

${ }^{1}$ Department of Anesthesiology and Reanimation, Faculty of Medicine, Canakkale Onsekiz Mart University, Canakkale, Turkey, ${ }^{2}$ Department of Pathology, Faculty of Medicine, Mersin University, Mersin, Turkey, ${ }^{3}$ Department of Anesthesiology and Reanimation, Faculty of Medicine, Dokuz Eylül University, Izmir, Turkey, and ${ }^{4}$ Department of Physiology, Faculty of Medicine, Canakkale Onsekiz Mart University, Canakkale, Turkey

Address for correspondence: H.A. Kiraz, MD, Canakkale Onsekiz Mart University, Faculty of Medicine, Department of Anesthesiology and Reanimation, Cumhuriyet Mahallesi Sahil Yolu No: 5 Anesteziyoloji ve Reanimasyon AD, 1. Kat Ameliyathane, Kepez, Canakkale, Turkey, Postcode: 17110.

Phone: +905427117551, Fax: +902862635957 small arteries, hemolysis secondary to intima damage and platelet aggregation. Apart from these events there is stasis, thrombosis and direct cytotoxicity in the vessel. Tissue damage is essentially determined by the chemical structure and amount of the medication (5). The pathogenesis of gangrene formation after intra-arterial injection of medication is not clear. To research the pathological process of intra-arterial injection the rabbit ear model is a good alternative. While there are visible differences to the human ear, it is helpful to observe the tissue response to intra-arterial medications (6).

Sugammadex is a medication with cyclodextrin structure that selectively binds to non-depolarizing muscle relaxants with aminosteroid structure like rocuronium and ends their activity. It shows high selectivity for rocuronium and vecuronium. The interaction with other anesthetic agents is relatively lower (7).

This study aims to investigate the histopathological effects that may form after intra-arterial application of two different doses of sugammadex used in clinical practice.

\section{Materials and methods}

The study was completed at Canakkale Onsekiz Mart University Experimental Research Center (COMUDAM) after receiving permission from Canakkale Onsekiz Mart University Animal Experiments Ethics Committee (Date of meeting: 27.03.2014 Decision No. 2014/04-02). The research used 15 adult male New Zealand white rabbits weighing from $3-5 \mathrm{~kg}$. Our study used the rabbit ear model described by Kinmonth and Shepherd (8). The 


\section{5-298}

animals were anesthetized after 6 hours of starvation and no access to water. Sterile techniques were used and the experimental group rabbits were once given $35 \mathrm{mg} / \mathrm{kg}$ ketamine $-5 \mathrm{mg} / \mathrm{kg}$ xylasine in the quadriceps femoris muscle of the rear leg by intramuscular (IM) route. If the single injection was above $2.5 \mathrm{~mL}$, it was divided in two and given to the right and left quadriceps femoris muscles separately. To show changes in response to anesthetic medication in animals the depth of anesthesia was determined by checking the palpebral or corneal reflexes. The front and rear extremities of all rabbits were held in crocodile electrodes, the areas were shaved and DII derivation ECG and pulse monitoring were done. During the experiment all experimental animals were given $0.9 \% \mathrm{NaCl}$ $40 \mathrm{~mL} / \mathrm{hr}$ infusion.

Rabbits were randomly divided into 3 groups:

1. Group $\mathrm{C}$ (Control, $\mathrm{n}=5)$ : The rabbits were given vein and artery catheterization, and $1 \mathrm{~mL}$ saline bolus was given into the rabbit ear central artery over 10 seconds.

2. Group Sgdx2 (Sugammadex, $n=5$ ): The rabbits were given vein and artery catheterization, and $2 \mathrm{mg} / \mathrm{kg}$ sugammadex bolus was given into the rabbit ear central artery for over 10 seconds.

3. Group Sgdx 16 (Sugammadex, $n=5$ ): The rabbits were given vein and artery catheterization, and $16 \mathrm{mg} / \mathrm{kg}$ sugammadex bolus was given into the rabbit ear central artery for over 10 seconds.

Rabbits with sufficient anesthesia first had venous catheterization of the ear veins with $26 \mathrm{G}$ branula; then intra-arterial intervention was realized with a $24 \mathrm{G}$ branula. As described in a previous study, all animals had Rabbit V-gel ${ }^{\circledR}$ inserted and oxygenation was supported with a mechanical ventilation device with respiration count held to 40/minute appropriate for rabbit physiology with a mix of $50 \%$ oxygen and $50 \%$ air for airway reliability (9). Body temperature was measured with a digital thermometer rectally inserted. During the full period of anesthesia, the rabbits were placed on a blanket heated up to $37-39^{\circ} \mathrm{C}$. The control group was given $1 \mathrm{~mL}$ saline bolus into the central artery of the ear. The final two groups were given 2 and $16 \mathrm{mg} / \mathrm{kg}$ sugammadex, respectively, intra-arterially. Three days later, all rabbits from the groups were given $35 \mathrm{mg} / \mathrm{kg}$ ketamine $-5 \mathrm{mg} / \mathrm{kg}$ xylasine (IM) and had the ear amputated under anesthesia for histopathological investigation.

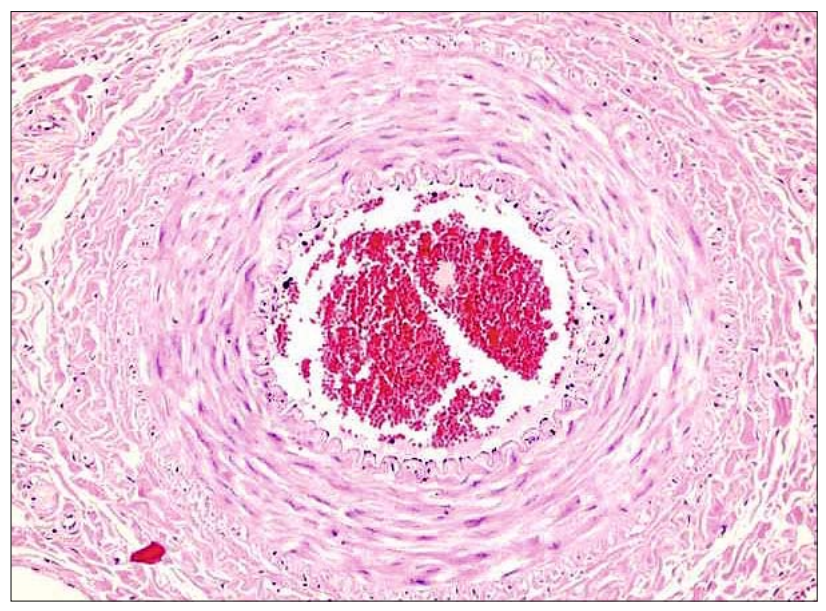

Fig. 1. Arterial structure in normal limits X400 magnification (H\&E).

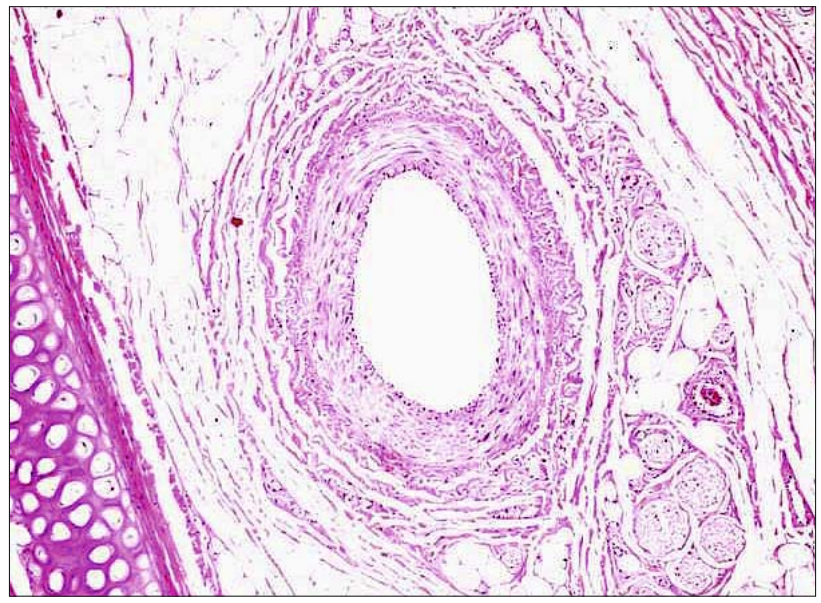

Fig. 2. Arterial structure in normal limits accompanied by very slight edema X200 magnification (H\&E).

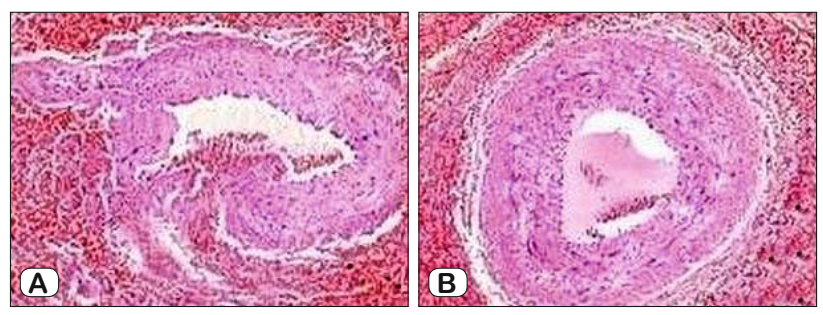

Fig. 3. A) Arterial wall damage, perivascular extravasation of erythrocytes. B) Single-cell necrosis of cell walls, perivascular extravasation of erythrocytes. X400 magnification (H\&E).

Sections made for macroscopic investigation, especially samples evaluated for arterial slices, were fixed in $10 \%$ buffered formaldehyde and immersed in paraffin before the paraffin block was serially sliced to $4-\mu \mathrm{m}$ thickness with a rotary microtome (Leica RM 2135, Leica Instruments, Nussloch, Germany). These sections were stained with hematoxylin-eosin (H\&E). The preparations were investigated under a light microscope (Nikon Eclipse E 600). Additionally, the obtained images were uploaded to a computer environment with the aid of a high resolution camera (Olympus DP-70, Japan).

\section{Statistical analysis}

Statistical analysis was performed by using the Statistical Package for the Social Sciences (SPSS) version 16.0 for Windows (SPSS Inc., Chicago, IL, USA). Mann-Whitney U test was used for statistical analysis. Descriptive statistics included Mean \pm Standard Deviation. A p value $<0.05$ was considered significant.

\section{Results}

Histopathological evaluation of the many samples of the material showed the arterial cross-sections were within normal limits in Group C (Fig. 1). In comparison to Group C and Group Sgdx16, the cross-sections belonging to Group $\mathrm{Sgdx} 2$ had slight edema but it was not statistically significant (Fig. 2). Notable changes were observed in the artery walls in the rabbit ear sections from 
Tab. 1. Histomorphological findings observed in the arterial walls of the study groups.

Groups Vascular Changes

Group C In normal limits

Sgdx2 Findings of slight edema

Sgdx16 Single-cell necrosis, necrotic debris, hemorrhage outside of arteries Sgdx - Sugammadex

Group Sgdx16. Arterial walls had findings of single-cell necrosis, necrotic debris and hemorrhage outside the artery linked to arterial damage (Fig. 3, Tab. 1). In Group Sgdx16, edema and single-cell necrosis were significantly higher when compared to Group $\mathrm{C}(\mathrm{p}$ $=0.008$ for both parameters). Single-cell necrosis was seen more significantly in Group Sgdx16 in comparison with Group Sgdx2 (p $=0.008)$. In terms of total histopathological scores of all groups, scores of Group Sgdx 16 were significantly higher when compared to Group C and Group $\operatorname{Sgdx} 2$ ( $\mathrm{p}=0.008$ for both parameters). The histological evaluation of all groups are shown in Table 2.

\section{Discussion}

Accidental application of intravenous medications used in anesthetic procedures through the intra-arterial route may result in local ischemia and following tissue necrosis (10). Accidental intra-arterial applications of anesthetic agents were reported and it proves to may be of such events (11). In literature, the effects of intra-arterial application of anesthetic materials such as thiopental, propofol, dexmedetomidine, fentanyl, pancuronium, ketamine, atracurium, rocuronium, midazolam, morphine and succinylcholine were researched $(4-8,10,12-15)$. However, there are limited data in literature about the intra-arterial application of sugammadex, selectively used to reverse the effects of systemic muscle relaxants in general anesthetic practice.

Neuromuscular blockers have long been used in anesthetic practice. Many medications have been developed to reverse the effects of these medications; a variety of medications such as neostigmine, suramin, purified human plasma cholinesterase, and cysteine have been used (16). Cyclodextrins take an important place in our daily life as constituents of food products, cosmetics, textiles and various medical products. The common point of all cyclodextrins is that they are water-soluble (17). Sugammadex is a modified form of gamma-cyclodextrin. Its effect is to form 1:1 water soluble complexes with steroid-structure neuromuscular blockers, and quickly remove the medication from the neuromuscular junction into plasma. Within this group of medications, it is most selective for rocuronium. The effectiveness is linked to dose with $2-16 \mathrm{mg} / \mathrm{kg}$ doses used in anesthetic practice (16). As a result, histopathological effects of 2 and $16 \mathrm{mg} / \mathrm{kg}$ doses of intra-arterial sugammadex were investigated in our study.

While gangrene has been observed with some medications in a variety of studies of intra-arterial medication application in humans, it has not been observed with others. The common characteristic of medications causing gangrene is that they are soluble in lipids and the pathological changes observed have some similarities. This supports the idea that the lipid solubility of a medication is a factor increasing the potential to cause gangrene. Medications highly soluble in membranes may be hemolytic, known to cause rapid lysis of erythrocytes at high concentrations (6). It has been proven in vitro that they have the same effect on other types of cells, not just erythrocytes (18). If medications soluble in membranes are given intra-arterially, they can reach high concentrations in the plasma membranes of vascular endothelial cells in a short time, starting a lytic process that may extend to intravascular thrombosis and gangrene. Additionally, it was shown that intraarterial injection of water-insoluble agents is associated with vascular complications (11). To investigate the pathological process of intra-arterial injections the rabbit ear model is a good alternative. Knill and Evans (6) used the rabbit ear model to investigate the effects of intra-arterial thiopental, chlorpromazine and amphetamine and found that medication doses causing gangrene in humans had a similar effect on rabbit ears. As a result, the rabbit ear model was selected for our study.

There are various theories about the cause of gangrene in tissues after intra-arterial medication. For example, some studies have reported theories such as acute obstruction of blood vessels, formation of insoluble acid crystals when high concentrations of medication mix with blood, direct irritation of vessel walls, vasoconstriction mediated by norepinephrine, arterial thrombosis, endothelial inflammation, venous constriction, solubility in lipids and high osmolarity $(3,6,8)$. In spite of all these theories, the underlying pathological mechanism has still not been clearly defined (3, 19). In this study, while no histopathological effect was observed in the control group and low-dose $(2 \mathrm{mg} / \mathrm{kg}$ ) sugammadex group, the necrotic changes observed in the high-dose $(16 \mathrm{mg} / \mathrm{kg})$ group lead us to consider that the effect is linked to the dose used. In a previous study, Hanci et al (20) administered sugammadex intraarterially at a dose of $4 \mathrm{mg} / \mathrm{kg}$ in rabbits and they reported significant histopathological changes in arterial endothelial structure due to endothelial damage compared to the control group. Hence, we decided to investigate the effects of sugammadex doses of 2 $\mathrm{mg} / \mathrm{kg}$ and $16 \mathrm{mg} / \mathrm{kg}$ to create a dose-response curve. Therefore, we think that the histopathological changes were dose-dependent. Additionally, when it is considered that the tissue damage is essentially determined by the chemical structure and amount of the

Tab. 2. Histological evaluation of the study groups (Mean \pm Standard Deviation).

\begin{tabular}{lcccc}
\hline Groups & Edema & Single-cell Necrosis & Necrotic Debris & Hemorrhage outside the Artery \\
\hline $\mathrm{C}(\mathrm{n}=5)$ & $0.00 \pm 0.00$ & $0.00 \pm 0.00$ & $0.00 \pm 0.00$ & $0.00 \pm 0.00$ \\
$\operatorname{Sgdx} 2(\mathrm{n}=5)$ & $0.60 \pm 0.54$ & $0.00 \pm 0.00$ & $0.00 \pm 0.00$ & $0.00 \pm 0.00$ \\
$\operatorname{Sgdx} 16(\mathrm{n}=5)$ & $1.00 \pm 0.00^{*}$ & $1.00 \pm 0.00^{* \dagger}$ & $0.40 \pm 0.54$ & $0.60 \pm 0.54$ \\
\hline
\end{tabular}

$*: \mathrm{p}<0.05$, in comparison with Group C, Mann-Whitney U test, $\uparrow: \mathrm{p}<0.05$, in comparison with Group Sgdx2, Mann-Whitney U test, $\ddagger$ : $\mathrm{p}<0$.05, in comparison with Group C, Mann-Whitney U test, \#: p $<0.05$, in comparison with Group Sgdx2, Mann-Whitney U test 


\section{5-298}

medication, it is not surprising that water-soluble sugammadex with a known chemical structure causes pathological changes only at high dose $(5,21)$.

\section{Conclusions}

In conclusion, being broadly used in anesthesia, sugammadex should be administered with care because when given by intraarterial route it may cause tissue damage and tissue loss and the patients' arteries should be carefully checked. Intravenous cannulation should be done by healthcare professionals with paying more attention to avoid intra-arterial injections. Further experimental research is required to understand the histopathological changes caused by sugammadex more clearly.

\section{References}

1. Prabhu R, Shenoy R, Thinda N, Patel A, Sadhu S. Be careful with an IV line. J Clin Diagn Res 2014; 8 (3): 166-167.

2. Kadlic T, Jacz K, Meskova M, Miklas R, Somodi J, Moravec R. Injuries of extremities resulting from accidental intra-arterial injections. Bratisl Lek Listy 1965; 45: 414-419.

3. Sen S, Chini EN, Brown MJ. Complications after unintentional intraarterial injection of drugs: risks, outcomes and management strategies. Mayo Clin Proc 2005; 80 (6): 783-795.

4. Lake $\mathbf{C}$, Beecroft $\mathbf{C L}$. Extravasation injuries and accidental intra-arterial injection. Contin Educ Anaesth Crit Care Pain 2010; 10 (4): 109-113.

5. Kilinç H, Esen O, Kahraman $\mathbf{H}$ et al. Experimental animal model of implementation of thiopental intraarterial papaverine pentoxifylline and treatment of damage incurred as a result of vessel wall histopathological evaluation. Medical Journal of Kocaeli 2013; 2: 10-18.

6. Knill RL, Evans D. Pathogenesis of gangrene following intra-arterial injection of drugs: a new hypothesis. Can Anaesth Soc J 1975; 22 (6): 637-646.

7. Welliver M. New drug sugammadex: a selective relaxant binding agent. AANA J 2006; 74 (5): 357-363.

8. Kinmonth JB, Shepherd RC. Accidental injection of thiopentone into arteries: studies of pathology and treatment. Br Med J 1959; 2 (5157): 914-918.
9. Toman H, Erbas M, Kiraz HA, Sahin H, Ovali MA, Uzun M. Comparison of effects of classic LMA, CobraPLA and V-Gel rabbit on hemodynamic response and QTc interval. Bratisl Lek Listy 2015; 116 (10): 632-636. DOI: 10.4149/BLL_2015_122).

10. Ghouri AF, Mading W, Prabaker K. Accidental intraarterial drug injections via intravascular catheters placed on the dorsum of the hand. Anesth Analg 2002; 95 (2): 487-491.

11. Jain A, Sahni N, Banik S, Solanki SL. Accidental intra-arterial injection of neostigmine with glycopyrrolate or atropine for reversal of residual neuromuscular blockade: a report of two cases. Anesth Analg 2012; 115 (1): 210-211.

12. Babacan A, Akçabay M, Uluoğlu O, Ozkoçak I, Karadenizli Y. The histopathological comparison of the results of the intra-arterial injections of propofol and thiopentone in rabbits. Mater Med Pol 1992; 24 (1): 41-44.

13. Ghatak T, Samanta S. Accidental intra-arterial dexmedetomidine injection in postoperative ward. Anaesth Intensive Care 2013; 41 (3): 431.

14. Righini M, Angellillo-Scherrer A, Gueddi S, Le Gal G, Bounameaux $\mathrm{H}$. Management of severe ischemia of the hand following intra-arterial injection. Thromb Haemost 2005; 94 (1): 219-221.

15. Fikkers BG, Wuis EW, Wijnen MH, Scheffer GJ. Intraarterial injection of anesthetic drugs. Anesth Analg 2006; 103 (3): 792-794.

16. Naguib M. Sugammadex: another milestone in clinical neuromuscular pharmacology. Anesth Analg 2007; 104 (3): 575-581.

17. Kurkov SV, Loftsson T. Cyclodextrins. Int J Pharm 2013; 453 (1): 167-180.

18. Ruutu T, Collan Y. Short-term effect of chlorpromazine on human leukocytes and erythrocytes. An electron microscopic study. Ann Med Exp Biol Fenn 1972; 50 (1): 42-48.

19. Hanci V, Yurtlu SB, Bilir S, Ayoğlu H, Demircan N, Özkoçak Turan I. Successful treatment for intra-arterial injection of pheniramine: case report. Türkiye Klinikleri J Anest Reanim 2009; 7 (1): 40-43.

20. Hanci V, Özbilgin Ş, Özbal S et al. Evaluation of the effects of intra-arterial sugammadex and dexmedetomidine: an experimental study. (Article in Press, Braz J Anesthesiol, DOI: 10.1016/j.bjane.2015.01.003).

21. Gijsenbergh F, Ramael S, Houwing N, van Iersel T. First human exposure of Org 25969, a novel agent to reverse the action of rocuronium bromide. Anesthesiology 2005; 103 (4): 695-703.

Received July 23, 2015. Accepted August 11, 2015. 